Open Access

\title{
Fekete-Szegő inequalities for certain class of analytic functions connected with $q$-analogue of Bessel function
}

\author{
Sheza M. El-Deeb ${ }^{1,2^{*}}$ (1) and Teodor Bulboacă ${ }^{3}$ (D)
}

\section{${ }^{*}$ Correspondence:}

shezaeldeeb@yahoo.com

1 Department of Mathematics,

Faculty of Science, Damietta

University, New Damietta 34517,

Egypt

${ }^{2}$ Department of Mathematics,

Faculty of Science and Arts in

Badaya, Al-Qassim University, Al-

Badaya, Al-Qassim, Kingdom of

Saudi Arabia

Full list of author information is

available at the end of the article

\begin{abstract}
In this paper, we obtain Fekete-Szegő inequalities for a certain class of analytic functions $f$ satisfying $1+\frac{1}{\zeta}\left[\frac{z\left(\mathcal{N}_{v, q}^{\lambda} f(z)\right)^{\prime}}{(1-\gamma) \mathcal{N}_{v, q}^{\lambda} f(z)+\gamma z\left(\mathcal{N}_{v, q}^{\lambda} f(z)\right)^{\prime}}-1\right] \prec \Psi(z)$. Application of our results to certain functions defined by convolution products with a normalized analytic function is given, and in particular, Fekete-Szegő inequalities for certain subclasses of functions defined through Poisson distribution are obtained.
\end{abstract}

Keywords: Fekete-Szegő inequality, Differential subordination, Bessel function of first kind, q-derivative, Poisson distribution

2010 Mathematics Subject Classification: 30C45, 30C80

\section{Introduction}

Let $\mathcal{A}$ denote the class of analytic functions of the form:

$$
f(z)=z+\sum_{k=2}^{\infty} a_{k} z^{k}, z \in \mathbb{D}:=\{z \in \mathbb{C}:|z|<1\},
$$

and $\mathcal{S}$ be the subclass of $\mathcal{A}$ which are univalent functions in $\mathbb{D}$.

If $k \in \mathcal{A}$ is given by:

$$
k(z)=z+\sum_{k=2}^{\infty} b_{k} z^{k}, z \in \mathbb{D},
$$

then, the Hadamard (or convolution) product of $f$ and $k$ is defined by:

$$
(f \times k)(z):=z+\sum_{k=2}^{\infty} a_{k} b_{k} z^{k}, z \in \mathbb{D} .
$$

If $f$ and $F$ are analytic functions in $\mathbb{D}$, we say that $f$ is subordinate to $F$, written $f \prec F$, if there exists a Schwarz function $w$, which is analytic in $\mathbb{D}$, with $w(0)=0$, and $|w(z)|<1$ for all $z \in \mathbb{D}$, such that $f(z)=F(w(z)), z \in \mathbb{D}$. Furthermore, if the function $F$ is univalent in $\mathbb{D}$, then we have the following equivalence (see [1] and [2] ):

$$
f(z) \prec F(z) \Leftrightarrow f(0)=F(0) \text { and } f(\mathbb{D}) \subset F(\mathbb{D}) .
$$

(c) The Author(s). 2019 Open Access This article is distributed under the terms of the Creative Commons Attribution 4.0 International License (http://creativecommons.org/licenses/by/4.0/), which permits unrestricted use, distribution, and reproduction in any medium, provided you give appropriate credit to the original author(s) and the source, provide a link to the Creative Commons license, and indicate if changes were made. 
The Bessel function of the first kind of order $v$ is defined by the infinite series:

$$
J_{v}(z):=\sum_{k \geq 0} \frac{(-1)^{k}\left(\frac{z}{2}\right)^{2 k+v}}{k ! \Gamma(k+v+1)}, z \in \mathbb{C}, \quad(v \in \mathbb{R}),
$$

where $\Gamma$ stands for the Gamma function. Recently, Szász and Kupán [3] investigated the univalence of the normalized Bessel function of the first kind $g_{v}: \mathbb{D} \rightarrow \mathbb{C}$ defined by (see also [4-6])

$$
\begin{array}{r}
g_{v}(z):=2^{v} \Gamma(v+1) z^{1-\frac{v}{2}} J_{v}\left(z^{\frac{1}{2}}\right) \\
=z+\sum_{k=2}^{\infty} \frac{(-1)^{k-1} \Gamma(v+1)}{4^{k-1}(k-1) ! \Gamma(k+v)} z^{k}, z \in \mathbb{D}, \quad(v \in \mathbb{R}) .
\end{array}
$$

For $0<q<1$, the $q$-derivative operator for $g_{\nu}$ is defined by:

$$
\begin{array}{r}
\partial_{q} g_{v}(z)=\partial_{q}\left[z+\sum_{k=2}^{\infty} \frac{(-1)^{k-1} \Gamma(v+1)}{4^{k-1}(k-1) ! \Gamma(k+v)} z^{k}\right]:=\frac{g_{v}(q z)-g_{v}(z)}{z(q-1)}= \\
1+\sum_{k=2}^{\infty} \frac{(-1)^{k-1} \Gamma(v+1)}{4^{k-1}(k-1) ! \Gamma(k+v)}[k, q] z^{k-1}, z \in \mathbb{D},
\end{array}
$$

where

$$
[k, q]:=\frac{1-q^{k}}{1-q}=1+\sum_{j=1}^{k-1} q^{j}, \quad[0, q]:=0 .
$$

Using definition formula (4), we will define the next two products:

(i) For any non-negative integer $k$, the $q$-shifted factorial is given by:

$$
[k, q]:=\left\{\begin{array}{lr}
1, & \text { if } k=0, \\
{[1, q][2, q][3, q] \ldots[k, q],} & \text { if } k \in \mathbb{N} .
\end{array}\right.
$$

(ii) For any positive number $r$, the q-generalized Pochhammer symbol is defined by:

$$
[r, q]_{k}:= \begin{cases}1, & \text { if } k=0 \\ {[r, q][r+1, q] \ldots[r+k-1, q],} & \text { if } k \in \mathbb{N} .\end{cases}
$$

For $v>0, \lambda>-1$, and $0<q<1$, define the function $\mathcal{I}_{v, q}^{\lambda}: \mathbb{D} \rightarrow \mathbb{C}$ by:

$$
\mathcal{I}_{\nu, q}^{\lambda}(z):=z+\sum_{k=2}^{\infty} \frac{(-1)^{k-1} \Gamma(v+1)}{4^{k-1}(k-1) ! \Gamma(k+v)} \frac{[k, q] !}{[\lambda+1, q]_{k-1}} z^{k}, z \in \mathbb{D} .
$$

Remark 1 A simple computation shows that:

$$
\mathcal{I}_{v, q}^{\lambda}(z) \times \mathcal{M}_{q, \lambda+1}(z)=z \partial_{q} g_{v}(z), z \in \mathbb{D},
$$

where the function $\mathcal{M}_{q, \lambda+1}$ is given by:

$$
\mathcal{M}_{q, \lambda+1}(z):=z+\sum_{k=2}^{\infty} \frac{[\lambda+1, q]_{k-1}}{[k-1, q] !} z^{k}, z \in \mathbb{D} .
$$


Using the definition of $q$-derivative along with the idea of convolutions, we introduce the linear operator $\mathcal{N}_{v, q}^{\lambda}: \mathcal{A} \rightarrow \mathcal{A}$ defined by:

$$
\begin{array}{r}
\mathcal{N}_{v, q}^{\lambda} f(z):=\mathcal{I}_{v, q}^{\lambda}(z) \times f(z)=z+\sum_{k=2}^{\infty} \psi_{k} a_{k} z^{k}, z \in \mathbb{D}, \\
(v>0, \lambda>-1,0<q<1),
\end{array}
$$

where

$$
\psi_{k}:=\frac{(-1)^{k-1} \Gamma(v+1)}{4^{k-1}(k-1) ! \Gamma(k+v)} \cdot \frac{[k, q] !}{[\lambda+1, q]_{k-1}} .
$$

Remark 2 From definition relation (5), we can easily verify that the next relations hold for all $f \in \mathcal{A}$ :

(i) $[\lambda+1, q] \mathcal{N}_{\nu, q}^{\lambda} f(z)=[\lambda, q] \mathcal{N}_{\nu, q}^{\lambda+1} f(z)+q^{\lambda} z \partial_{q}\left(\mathcal{N}_{\nu, q}^{\lambda+1} f(z)\right), z \in \mathbb{D}$;

(ii) $\lim _{q \rightarrow 1^{-}} \mathcal{N}_{v, q}^{\lambda} f(z)=\mathcal{I}_{v, 1}^{\lambda} \times f(z)=: \mathcal{I}_{v}^{\lambda} f(z)=$

$z+\sum_{k=2}^{\infty} \frac{k !}{(\lambda+1)_{k-1}} \frac{(-1)^{k-1} \Gamma(v+1)}{4^{k-1}(k-1) ! \Gamma(k+v)} a_{k} z^{k}, z \in \mathbb{D}$.

Now, we define the class of functions $\mathcal{M}_{v, q}^{\lambda, \gamma}(\zeta ; \Psi)$ as follows:

Definition 1 Let $\Psi(z):=1+B_{1} z+B_{2} z^{2}+\ldots, z \in \mathbb{D}$, with $B_{1}>0$, be a starlike (univalent) function with respect to 1 , which maps the unit disk $\mathbb{D}$ onto a region included in the right half plane which is symmetric with respect to the real axis. For $\zeta \in \mathbb{C}^{*}$, and $0 \leq \gamma<1$, the function $f \in \mathcal{A}$ is said to be in the class $\mathcal{M}_{\nu, q}^{\lambda, \gamma}(\zeta ; \Psi)$ if the function

$$
1+\frac{1}{\zeta}\left[\frac{z\left(\mathcal{N}_{\nu, q}^{\lambda} f(z)\right)^{\prime}}{(1-\gamma) \mathcal{N}_{\nu, q}^{\lambda} f(z)+\gamma z\left(\mathcal{N}_{\nu, q}^{\lambda} f(z)\right)^{\prime}}-1\right]
$$

is analytic in $\mathbb{D}$ and satisfies:

$$
\begin{aligned}
1+\frac{1}{\zeta} & {\left[\frac{z\left(\mathcal{N}_{v, q}^{\lambda} f(z)\right)^{\prime}}{(1-\gamma) \mathcal{N}_{\nu, q}^{\lambda} f(z)+\gamma z\left(\mathcal{N}_{\nu, q}^{\lambda} f(z)\right)^{\prime}}-1\right] \prec \Psi(z) } \\
& \left(v>0, \lambda>-1,0<q<1, \zeta \in \mathbb{C}^{*}, 0 \leq \gamma<1\right) .
\end{aligned}
$$

Putting $q \rightarrow 1^{-}$, we obtain that $\lim _{q \rightarrow 1^{-}} \mathcal{M}_{v, q}^{\lambda, \gamma}(\zeta ; \Psi)=: \mathcal{G}_{v}^{\lambda, \gamma}(\zeta ; \Psi)$, where

$$
\begin{array}{r}
\mathcal{G}_{v}^{\lambda, \gamma}(\zeta ; \Psi):=\left\{1+\frac{1}{\zeta}\left[\frac{z\left(\mathcal{I}_{\nu}^{\lambda} f(z)\right)^{\prime}}{(1-\gamma) \mathcal{I}_{\nu}^{\lambda} f(z)+\gamma z\left(\mathcal{I}_{\nu}^{\lambda} f(z)\right)^{\prime}}-1\right] \prec \Psi(z)\right\} \\
\left(v>0, \lambda>-1, \zeta \in \mathbb{C}^{*}, 0 \leq \gamma<1\right) .
\end{array}
$$

In this paper, we obtain the Fekete-Szegö inequalities for the functions of the class $\mathcal{M}_{\nu, q}^{\lambda, \gamma}(\zeta ; \Psi)$. We give some application of our results to certain functions defined by convolution products with a normalized analytic function. In particular, Fekete-Szegő inequalities for certain subclasses of functions defined through Poisson distribution are obtained. 


\section{Fekete-Szegő problem}

Denoted by $\mathcal{P}$, the well-known Carathéodory's class of analytic functions in $\mathbb{D}$, normalized with $P(0)=1$, and having positive real part in $\mathbb{D}$, that is $\operatorname{Re} P(z)>0$ for all $z \in \mathbb{D}$ (see [7]).

To prove our results, we need the following two lemmas.

Lemma $1\left[8\right.$, Lemma 3] If $p(z)=1+c_{1} z+c_{2} z^{2}+\cdots \in \mathcal{P}$, and $\alpha$ is a complex number, then

$$
\max \left|c_{2}-\alpha c_{1}^{2}\right|=2 \max \{1 ;|2 \alpha-1|\} .
$$

Lemma 2 [9, Lemma 1] If $p(z)=1+c_{1} z+c_{2} z^{2}+\cdots \in \mathcal{P}$, then

$$
\left|c_{2}-\alpha c_{1}^{2}\right| \leq \begin{cases}-4 \alpha+2, & \text { if } \alpha \leq 0 \\ 2, & \text { if } 0 \leq \alpha \leq 1 \\ 4 \alpha-2, & \text { if } \alpha \geq 1\end{cases}
$$

When $\alpha<0$ or $\alpha>1$, the equality holds if and only if $p(z)=\frac{1+z}{1-z}$ or one of its rotations. If $0<\alpha<1$, then the equality holds if and only if $p(z)=\frac{1+z^{2}}{1-z^{2}}$ or one of its rotations.

If $\alpha=0$, the equality holds if and only if:

$$
p(z)=\left(\frac{1}{2}+\frac{\lambda}{2}\right) \frac{1+z}{1-z}+\left(\frac{1}{2}-\frac{\lambda}{2}\right) \frac{1-z}{1+z}, \quad \text { with } \quad 0 \leq \lambda \leq 1,
$$

or one of its rotations.

If $\alpha=1$, the equality holds if and only if:

$$
\frac{1}{p(z)}=\left(\frac{1}{2}+\frac{\lambda}{2}\right) \frac{1+z}{1-z}+\left(\frac{1}{2}-\frac{\lambda}{2}\right) \frac{1-z}{1+z}, \quad \text { with } \quad 0 \leq \lambda \leq 1 .
$$

Like it was mentioned in [9, pages 162-163], although the above upper bound is sharp, it can be improved as follows when $0<\alpha<1$ :

$$
\left|c_{2}-\alpha c_{1}^{2}\right|+\alpha\left|c_{1}\right|^{2} \leq 2, \quad \text { if } \quad 0<\alpha \leq \frac{1}{2},
$$

and

$$
\left|c_{2}-\alpha c_{1}^{2}\right|+(1-\alpha)\left|c_{1}\right|^{2} \leq 2, \quad \text { if } \quad \frac{1}{2} \leq \alpha<1 .
$$

Theorem 1 If the function $f$ given by (1) belongs to the class $\mathcal{M}_{\nu, q}^{\lambda, \gamma}(\zeta ; \Psi)$, with $\Psi(z)=$ $1+B_{1} z+B_{2} z^{2}+\ldots$ satisfying the conditions of Definition 1 , and $\mu$ is a complex number, then:

$$
\left|a_{3}-\mu a_{2}^{2}\right| \leq \frac{|\zeta| B_{1}}{2(1-\gamma) \psi_{3}} \cdot \max \left\{1 ;\left|\frac{B_{2}}{B_{1}}+\frac{\zeta B_{1}(1+\gamma)}{1-\gamma}-\frac{2 \mu \zeta B_{1} \psi_{3}}{(1-\gamma) \psi_{2}^{2}}\right|\right\},
$$

where $\psi_{k}, k \in\{2,3\}$, are given by (6).

Proof If $f \in \mathcal{M}_{v, q}^{\lambda, \gamma}(\zeta ; \Psi)$, then there exists a Schwarz function $w$, that is $w$ is analytic in $\mathbb{D}$, with $w(0)=0$ and $|w(z)|<1, z \in \mathbb{D}$, such that:

$$
1+\frac{1}{\zeta}\left[\frac{z\left(\mathcal{N}_{\nu, q}^{\lambda} f(z)\right)^{\prime}}{(1-\gamma) \mathcal{N}_{\nu, q}^{\lambda} f(z)+\gamma z\left(\mathcal{N}_{\nu, q}^{\lambda} f(z)\right)^{\prime}}-1\right]=\Psi(w(z)), z \in \mathbb{D} .
$$

Since $w$ is a Schwarz function, it follows that the function $p_{1}$ defined by: 


$$
p_{1}(z):=\frac{1+w(z)}{1-w(z)}=1+c_{1} z+c_{2} z^{2}+\ldots, z \in \mathbb{D},
$$

belongs to $\mathcal{P}$. Defining the function $p$ by:

$$
p(z):=1+\frac{1}{\zeta}\left[\frac{z\left(\mathcal{N}_{\nu, q}^{\lambda} f(z)\right)^{\prime}}{(1-\gamma) \mathcal{N}_{\nu, q}^{\lambda} f(z)+\gamma z\left(\mathcal{N}_{\nu, q}^{\lambda} f(z)\right)^{\prime}}-1\right]=1+d_{1} z+d_{2} z^{2}+\ldots, z \in \mathbb{D},
$$

in view of (9) and (10), we have:

$$
p(z)=\Psi\left(\frac{p_{1}(z)-1}{p_{1}(z)+1}\right), z \in \mathbb{D} .
$$

From (10), we easily get:

$$
\frac{p_{1}(z)-1}{p_{1}(z)+1}=\frac{1}{2}\left[c_{1} z+\left(c_{2}-\frac{c_{1}^{2}}{2}\right) z^{2}+\left(c_{3}+\frac{c_{1}^{3}}{4}-c_{1} c_{2}\right) z^{3}+\ldots\right], z \in \mathbb{D} ;
$$

therefore,

$$
\Psi\left(\frac{p_{1}(z)-1}{p_{1}(z)+1}\right)=1+\frac{1}{2} B_{1} c_{1} z+\left[\frac{1}{2} B_{1}\left(c_{2}-\frac{c_{1}^{2}}{2}\right)+\frac{1}{4} B_{2} c_{1}^{2}\right] z^{2}+\ldots, z \in \mathbb{D},
$$

and from (12), we obtain:

$$
d_{1}=\frac{1}{2} B_{1} c_{1} \quad \text { and } \quad d_{2}=\frac{1}{2} B_{1}\left(c_{2}-\frac{c_{1}^{2}}{2}\right)+\frac{1}{4} B_{2} c_{1}^{2} .
$$

On the other hand, from (11), according to (5), it follows that

$$
d_{1}=\frac{(1-\gamma) a_{2} \psi_{2}}{\zeta} \quad \text { and } \quad d_{2}=\frac{2(1-\gamma) a_{3} \psi_{3}}{\zeta}-\frac{(1-\gamma)(1+\gamma) a_{2}^{2} \psi_{2}^{2}}{\zeta},
$$

and combining (13) with (14), we have:

$$
a_{2}=\frac{\zeta B_{1} c_{1}}{2(1-\gamma) \psi_{2}},
$$

and

$$
a_{3}=\frac{\zeta B_{1}}{4(1-\gamma) \psi_{3}}\left[c_{2}-\frac{c_{1}^{2}}{2}+\frac{1}{2} \frac{B_{2}}{B_{1}} c_{1}^{2}+\frac{\zeta B_{1}(1+\gamma) c_{1}^{2}}{2(1-\gamma)}\right] .
$$

Therefore,

$$
a_{3}-\mu a_{2}^{2}=\frac{\zeta B_{1}}{4(1-\gamma) \psi_{3}}\left(c_{2}-\alpha c_{1}^{2}\right)
$$

where

$$
\alpha=\frac{1}{2}\left[1-\frac{B_{2}}{B_{1}}-\frac{\zeta B_{1}(1+\gamma)}{1-\gamma}+\frac{2 \mu \zeta B_{1} \psi_{3}}{(1-\gamma) \psi_{2}^{2}}\right],
$$

and from Lemma 1 , our result follows immediately.

Putting $q \rightarrow 1^{-}$in Theorem 1, we obtain the next corollary:

Corollary 1 If the function $f$ given by (1) belongs to the class $\mathcal{G}_{v}^{\lambda, \gamma}(\zeta ; \Psi)$, with $\Psi(z)=$ $1+B_{1} z+B_{2} z^{2}+\ldots$ satisfying the conditions of Definition 1 , and $\mu$ is a complex number, then 


$$
\begin{array}{r}
\left|a_{3}-\mu a_{2}^{2}\right| \leq \\
\frac{8|\zeta| B_{1}(\lambda+1)_{2}(\nu+1)_{2}}{3(1-\gamma)} \cdot \max \left\{1 ;\left|\frac{B_{2}}{B_{1}}+\frac{\zeta B_{1}(1+\gamma)}{1-\gamma}-\frac{3 \mu \zeta B_{1}(\lambda+1)(\nu+1)}{2(1-\gamma)(\lambda+2)(v+2)}\right|\right\} .
\end{array}
$$

Using a similar proof like for Theorem 1 combined with Lemma 2, we can obtain the following theorem:

Theorem 2 If the function $f$ given by (1) belongs to the class $\mathcal{M}_{v, q}^{\lambda, \gamma}(\zeta ; \Psi)$, with $\Psi(z)=$ $1+B_{1} z+B_{2} z^{2}+\ldots$ satisfying the conditions of Definition 1 and $\mu, B_{2} \in \mathbb{R}$, and $\zeta>0$, then

$$
\left|a_{3}-\mu a_{2}^{2}\right| \leq \begin{cases}\frac{\zeta B_{1}}{2(1-\gamma) \psi_{3}}\left[\frac{B_{2}}{B_{1}}+\frac{\zeta B_{1}(1+\gamma)}{1-\gamma}-\frac{2 \mu \zeta B_{1} \psi_{3}}{(1-\gamma) \psi_{2}^{2}}\right], & \text { if } \mu \leq \sigma_{1}, \\ \frac{\zeta B_{1}}{2(1-\gamma) \psi_{3}}, & \text { if } \sigma_{1} \leq \mu \leq \sigma_{2}, \\ \frac{-\zeta B_{1}}{2(1-\gamma) \psi_{3}}\left[\frac{B_{2}}{B_{1}}+\frac{\zeta B_{1}(1+\gamma)}{1-\gamma}-\frac{2 \mu \zeta B_{1} \psi_{3}}{(1-\gamma) \psi_{2}^{2}}\right], & \text { if } \mu \geq \sigma_{2},\end{cases}
$$

with

$$
\sigma_{1}=\frac{(1-\gamma) \psi_{2}^{2}}{2 \zeta B_{1} \psi_{3}}\left[-1+\frac{B_{2}}{B_{1}}+\frac{\zeta B_{1}(1+\gamma)}{1-\gamma}\right],
$$

and

$$
\sigma_{2}=\frac{(1-\gamma) \psi_{2}^{2}}{2 \zeta B_{1} \psi_{3}}\left[1+\frac{B_{2}}{B_{1}}+\frac{\zeta B_{1}(1+\gamma)}{1-\gamma}\right],
$$

where $\psi_{k}, k \in\{2,3\}$, are given by $(6)$.

Proof With the same proof like those of Theorem 1, we obtain the equalities (16) and (17) hold.

(i) According to the first part of Lemma 2, we have:

$$
\left|c_{2}-\alpha c_{1}^{2}\right| \leq-4 \alpha+2 \text {, if } \alpha \leq 0 .
$$

Using (17), simple computation shows that the inequality $\alpha \leq 0$ is equivalent to $\mu \leq \sigma_{1}$, and from (16) combined with the inequality $\left|c_{2}-\alpha c_{1}^{2}\right| \leq-4 \alpha+2$, the first of our theorem is proved.

(ii) The second part of Lemma 2 shows that:

$$
\left|c_{2}-\alpha c_{1}^{2}\right| \leq 2 \text {, if } 0 \leq \alpha \leq 1 .
$$

From (17), it is easy to check that the inequality $0 \leq \alpha \leq 1$ is equivalent to $\sigma_{1} \leq \mu \leq \sigma_{2}$. From the relation (16), the inequality $\left|c_{2}-\alpha c_{1}^{2}\right| \leq 2$ proves the second part of our result.

(iii) Finally, form the third part of Lemma 2, we have:

$$
\left|c_{2}-\alpha c_{1}^{2}\right| \leq 4 \alpha-2 \text {, if } \alpha \geq 1 \text {. }
$$

The relation (17) shows immediately that $\alpha \geq 1$ is equivalent to $\mu \geq \sigma_{2}$, while (16) combined with the inequality $\left|c_{2}-\alpha c_{1}^{2}\right| \leq 4 \alpha-2$ proves the last part of our result.

Taking $q \rightarrow 1^{-}$in Theorem 2 , we get the next special case:

Corollary 2 If the function f given by (1) belongs to the class $\mathcal{G}_{v}^{\lambda, \gamma}(\zeta ; \Psi)$, with $\Psi(z)=$ $1+B_{1} z+B_{2} z^{2}+\ldots$ satisfying the conditions of Definition 1 and $\mu, B_{2} \in \mathbb{R}$, and $\zeta>0$, then 


$$
\left|a_{3}-\mu a_{2}^{2}\right| \leq\left\{\begin{array}{rr}
\frac{8 \zeta B_{1}(\lambda+1)_{2}(v+1)_{2}}{3(1-\gamma)}\left[\frac{B_{2}}{B_{1}}+\frac{\zeta B_{1}(1+\gamma)}{1-\gamma}-\frac{3 \mu \zeta B_{1}(\lambda+1)(v+1)}{2(1-\gamma)(\lambda+2)(v+2)}\right], \\
\text { if } \mu \leq \eta_{1}, \\
\frac{8 \zeta B_{1}(\lambda+1)_{2}(v+1)_{2},}{3(1-\gamma)}, \quad \text { if } \eta_{1} \leq \mu \leq \eta_{2}, \\
\frac{-8 \zeta B_{1}(\lambda+1)_{2}(v+1)_{2}}{3(1-\gamma)}\left[\frac{B_{2}}{B_{1}}+\frac{\zeta B_{1}(1+\gamma)}{1-\gamma}-\frac{3 \mu \zeta B_{1}(\lambda+1)(v+1)}{2(1-\gamma)(\lambda+2)(v+2)}\right], \\
\text { if } \mu \geq \eta_{2},
\end{array}\right.
$$

with

$$
\eta_{1}=\frac{2(1-\gamma)(\lambda+2)(v+2)}{3 \zeta B_{1}(\lambda+1)(v+1)}\left[-1+\frac{B_{2}}{B_{1}}+\frac{\zeta B_{1}(1+\gamma)}{(1-\gamma)}\right]
$$

and

$$
\eta_{2}=\frac{2(1-\gamma)(\lambda+2)(v+2)}{3 \zeta B_{1}(\lambda+1)(v+1)}\left[1+\frac{B_{2}}{B_{1}}+\frac{\zeta B_{1}(1+\gamma)}{(1-\gamma)}\right] .
$$

With a similar proof like for Theorem 1 and using the inequalities (7) and (8), we obtained the next result.

Theorem 3 If the function f given by (1) belongs to the class $\mathcal{M}_{\nu, q}^{\lambda, \gamma}(\zeta ; \Psi)$, with $\Psi(z)=$ $1+B_{1} z+B_{2} z^{2}+\ldots$ satisfying the conditions of Definition 1 and $\mu, B_{2} \in \mathbb{R}$, and $\zeta>0$, then the next inequalities hold:

(i) for $\sigma_{1}<\mu \leq \sigma_{3}$, we have

$$
\left|a_{3}-\mu a_{2}^{2}\right|+\frac{(1-\gamma) \psi_{2}^{2}}{2 \zeta B_{1} \psi_{3}}\left[1-\frac{B_{2}}{B_{1}}-\frac{\zeta B_{1}(1+\gamma)}{1-\gamma}+\frac{2 \mu \zeta B_{1} \psi_{3}}{(1-\gamma) \psi_{2}^{2}}\right]\left|a_{2}\right|^{2} \leq \frac{\zeta B_{1}}{2(1-\gamma) \psi_{3}} ;
$$

(ii) for $\sigma_{3} \leq \mu \leq \sigma_{2}$, we have

$$
\left|a_{3}-\mu a_{2}^{2}\right|+\frac{(1-\gamma) \psi_{2}^{2}}{2 \zeta B_{1} \psi_{3}}\left[1+\frac{B_{2}}{B_{1}}+\frac{\zeta B_{1}(1+\gamma)}{1-\gamma}-\frac{2 \mu \zeta B_{1} \psi_{3}}{(1-\gamma) \psi_{2}^{2}}\right]\left|a_{2}\right|^{2} \leq \frac{\zeta B_{1}}{2(1-\gamma) \psi_{3}},
$$

where $\sigma_{1}$ and $\sigma_{2}$ are defined by (18) and (19), respectively,

$$
\sigma_{3}=\frac{(1-\gamma) \psi_{2}^{2}}{2 \zeta B_{1} \psi_{3}}\left[\frac{B_{2}}{B_{1}}+\frac{\zeta B_{1}(1+\gamma)}{(1-\gamma)}\right]
$$

and $\psi_{k}, k \in\{2,3\}$, are given by $(6)$.

Proof With the same computations like in the proof of Theorem 1, we obtain the relations (16) and (17), while (15) is equivalent to:

$$
c_{1}=\frac{2(1-\gamma) \psi_{2}}{\zeta B_{1}}
$$

(i) To prove the first part of our theorem, we will use the inequality (7). Thus, according to (16), (17), and the above relation, it is easy to check that (7) could be written in the equivalent form (22), while the assumption $0<\alpha \leq \frac{1}{2}$ is equivalent to $\sigma_{1}<\mu \leq \sigma_{3}$.

(ii) For the proof of the second part of our result, we will use the inequality (8). From (16), (17), and (24), it follows that (8) could be written in the form (23), and the assumption $\frac{1}{2} \leq \alpha<1$ is equivalent to $\sigma_{3}<\mu \leq \sigma_{2}$. 
Putting $q \rightarrow 1^{-}$in Theorem 3 , we obtain the following result:

Corollary 3 If the function $f$ given by (1) belongs to the class $\mathcal{G}_{v}^{\lambda, \gamma}(\zeta ; \Psi)$, with $\Psi(z)=$ $1+B_{1} z+B_{2} z^{2}+\ldots$ satisfying the conditions of Definition 1 and $\mu, B_{2} \in \mathbb{R}$, and $\zeta>0$, then the next inequalities hold:

(i) for $\eta_{1}<\mu \leq \eta_{3}$, we have

$$
\begin{array}{r}
\left.\left.\mid \begin{array}{r}
\left|a_{3}-\mu a_{2}^{2}\right| \\
3 \zeta B_{1}(\lambda+1)(\nu+1)
\end{array}\right]+\frac{B_{2}}{B_{1}}-\frac{\zeta B_{1}(1+\gamma)}{1-\gamma}+\frac{3 \mu \zeta B_{1}(\lambda+1)(\nu+1)}{2(1-\gamma)(\lambda+2)(\nu+2)}\right]\left|a_{2}\right|^{2} \\
\leq \frac{8 \zeta B_{1}(\lambda+1)_{2}(\nu+1)_{2}}{3(1-\gamma)}
\end{array}
$$

(ii) for $\eta_{3} \leq \mu \leq \eta_{2}$, we have

$$
\begin{array}{r}
\left|a_{3}-\mu a_{2}^{2}\right| \\
+\frac{2(1-\gamma)(\lambda+2)(\nu+2)}{3 \zeta B_{1}(\lambda+1)(\nu+1)}\left[1+\frac{B_{2}}{B_{1}}+\frac{\zeta B_{1}(1+\gamma)}{1-\gamma}-\frac{3 \mu \zeta B_{1}(\lambda+1)(\nu+1)}{2(1-\gamma)(\lambda+2)(\nu+2)}\right]\left|a_{2}\right|^{2} \\
\leq \frac{8 \zeta B_{1}(\lambda+1)_{2}(\nu+1)_{2}}{3(1-\gamma)}
\end{array}
$$

where $\eta_{1}$ and $\eta_{2}$ are defined by (20) and (21), respectively, and

$$
\eta_{3}=\frac{2(1-\gamma)(\lambda+2)(v+2)}{3 \zeta B_{1}(\lambda+1)(v+1)}\left[\frac{B_{2}}{B_{1}}+\frac{\zeta B_{1}(1+\gamma)}{(1-\gamma)}\right] .
$$

\section{Applications to functions defined by poisson distribution}

In [10], Porwal studied a power series whose coefficients are probabilities of the Poisson distribution, that is:

$$
\mathrm{I}_{m}(z)=z+\sum_{k=2}^{\infty} \frac{m^{k-1}}{(k-1) !} e^{-m} z^{k}, z \in \mathbb{D}, \quad(m>0),
$$

and motivated by this investigation Srivastava and Porwal [11] introduced the linear operator $\mathcal{I}^{m}: \mathcal{A} \rightarrow \mathcal{A}$ defined by:

$$
\mathcal{I}^{m} f(z):=\mathrm{I}_{m}(z) \times f(z)=z+\sum_{k=2}^{\infty} \frac{m^{k-1}}{(k-1) !} e^{-m} a_{k} z^{k}, z \in \mathbb{D}
$$

where $f \in \mathcal{A}$ has the form (1).

Definition 2 Let the function $\Psi$ satisfying the conditions of Definition 1 . For $\zeta \in \mathbb{C}^{*}$, $0 \leq \gamma<1$, and $k \in \mathcal{A}$, the function $f \in \mathcal{A}$ is said to be in the class $\mathcal{M}_{\nu, q}^{\lambda, \gamma}(\zeta ; k ; \Psi)$ if $f \times k \in \mathcal{M}_{v, q}^{\lambda, \gamma}(\zeta ; \Psi)$, that is"

$$
1+\frac{1}{\zeta}\left[\frac{z\left(\mathcal{N}_{\nu, q}^{\lambda}(f \times k)(z)\right)^{\prime}}{(1-\gamma) \mathcal{N}_{\nu, q}^{\lambda}(f \times k)(z)+\gamma z\left(\mathcal{N}_{\nu, q}^{\lambda}(f \times k)(z)\right)^{\prime}}-1\right]
$$

is analytic in $\mathbb{D}$ and satisfies 


$$
\begin{array}{r}
1+\frac{1}{\zeta}\left[\frac{z\left(\mathcal{N}_{v, q}^{\lambda}(f \times k)(z)\right)^{\prime}}{(1-\gamma) \mathcal{N}_{\nu, q}^{\lambda}(f \times k)(z)+\gamma z\left(\mathcal{N}_{\nu, q}^{\lambda}(f \times k)(z)\right)^{\prime}}-1\right] \prec \Psi(z) \\
\left(v>0, \lambda>-1,0<q<1, \zeta \in \mathbb{C}^{*}, 0 \leq \gamma<1\right) .
\end{array}
$$

A special case of the class $\mathcal{M}_{v, q}^{\lambda, \gamma}(\zeta ; k ; \Psi)$ is obtained for $k=\mathrm{I}_{m}$; hence, $f \in$ $\mathcal{M}_{v, q}^{\lambda, \gamma}\left(\zeta ; \mathrm{I}_{m} ; \Psi\right)$ if and only if $\mathcal{I}^{m} f \in \mathcal{M}_{v, q}^{\lambda, \gamma}(\zeta ; \Psi)$.

Applying Theorems 1 and 2 for the function $f \times k$ given by (3), we get the following results, respectively:

Theorem 4 If the function fiven by (1) belongs to the class $\mathcal{M}_{v, q}^{\lambda, \gamma}(\zeta ; k ; \Psi)$, with $\Psi(z)=$ $1+B_{1} z+B_{2} z^{2}+\ldots, k \in \mathcal{A}$ is given by (2) with $b_{2} b_{3} \neq 0$, and $\mu$ is a complex number, then

$$
\left|a_{3}-\mu a_{2}^{2}\right| \leq \frac{|\zeta| B_{1}}{2(1-\gamma)\left|b_{3}\right| \psi_{3}} \cdot \max \left\{1,\left|\frac{B_{2}}{B_{1}}+\frac{\zeta B_{1}(1+\gamma)}{1-\gamma}-\frac{2 \mu \zeta B_{1} b_{3} \psi_{3}}{(1-\gamma) b_{2}^{2} \psi_{2}^{2}}\right|\right\},
$$

where $\psi_{k}$ and $k \in\{2,3\}$ are given by (6).

Theorem 5 If the function fiven by (1) belongs to the class $\mathcal{M}_{v, q}^{\lambda, \gamma}(\zeta ; k ; \Psi)$, with $\Psi(z)=$ $1+B_{1} z+B_{2} z^{2}+\ldots$ satisfying the conditions of Definition 1 and $\mu, B_{2} \in \mathbb{R}, k \in \mathcal{A}$ is given by (2) with $b_{2} b_{3} \neq 0$, and $\zeta>0$, then

$$
\left|a_{3}-\mu a_{2}^{2}\right| \leq \begin{cases}\frac{\zeta B_{1}}{2(1-\gamma)\left|b_{3}\right| \psi_{3}}\left[\frac{B_{2}}{B_{1}}+\frac{\zeta B_{1}(1+\gamma)}{(1-\gamma)}-\frac{2 \mu \zeta B_{1} b_{3} \psi_{3}}{(1-\gamma) b_{2}^{2} \psi_{2}^{2}}\right], & \text { if } \mu \leq \sigma_{1}, \\ \frac{\zeta B_{1}}{2(1-\gamma)\left|b_{3}\right| \psi_{3}}, & \text { if } \sigma_{1} \leq \mu \leq \sigma_{2}, \\ \frac{-\zeta B_{1}}{2(1-\gamma)\left|b_{3}\right| \psi_{3}}\left[\frac{B_{2}}{B_{1}}+\frac{\zeta B_{1}(1+\gamma)}{(1-\gamma)}-\frac{2 \mu \zeta B_{1} b_{3} \psi_{3}}{(1-\gamma) b_{2}^{2} \psi_{2}^{2}}\right], & \text { if } \mu \geq \sigma_{2},\end{cases}
$$

with

$$
\sigma_{1}=\frac{(1-\gamma) b_{2}^{2} \psi_{2}^{2}}{2 \zeta B_{1} b_{3} \psi_{3}}\left[-1+\frac{B_{2}}{B_{1}}+\frac{\zeta B_{1}(1+\gamma)}{1-\gamma}\right],
$$

and

$$
\sigma_{2}=\frac{(1-\gamma) b_{2}^{2} \psi_{2}^{2}}{2 \zeta B_{1} b_{3} \psi_{3}}\left[1+\frac{B_{2}}{B_{1}}+\frac{\zeta B_{1}(1+\gamma)}{1-\gamma}\right]
$$

and $\psi_{k}, k \in\{2,3\}$, are given by (6).

For $k:=\mathrm{I}_{m}$, we have

$$
b_{2}=m e^{-m} \quad \text { and } \quad b_{3}=\frac{m^{2}}{2} e^{-m},
$$

and for this special case from Theorems 4 and 5, we deduce to the following results, respectively:

Theorem 6 If the function $f$ given by (1) belongs to the class $\mathcal{M}_{v, q}^{\lambda, \gamma}\left(\zeta ; \mathrm{I}_{m} ; \Psi\right)$, with $\Psi(z)=1+B_{1} z+B_{2} z^{2}+\ldots$, and $\mu$ is a complex number, then

$$
\left|a_{3}-\mu a_{2}^{2}\right| \leq \frac{|\zeta| B_{1}}{(1-\gamma) m^{2} e^{-m} \psi_{3}} \cdot \max \left\{1 ;\left|\frac{B_{2}}{B_{1}}+\frac{\zeta B_{1}(1+\gamma)}{1-\gamma}-\frac{\mu \zeta B_{1} \psi_{3}}{(1-\gamma) e^{-m} \psi_{2}^{2}}\right|\right\}
$$


where $\psi_{k}$ and $k \in\{2,3\}$ are given by (6).

Theorem 7 If the function fgiven by (1) belongs to the class $\mathcal{M}_{v, q}^{\lambda, \gamma}\left(\zeta ; \mathrm{I}_{m} ; \Psi\right)$, with $\Psi(z)=$ $1+B_{1} z+B_{2} z^{2}+\ldots$ satisfying the conditions of Definition 1 and $\mu, B_{2} \in \mathbb{R}$, and $\zeta>0$, then

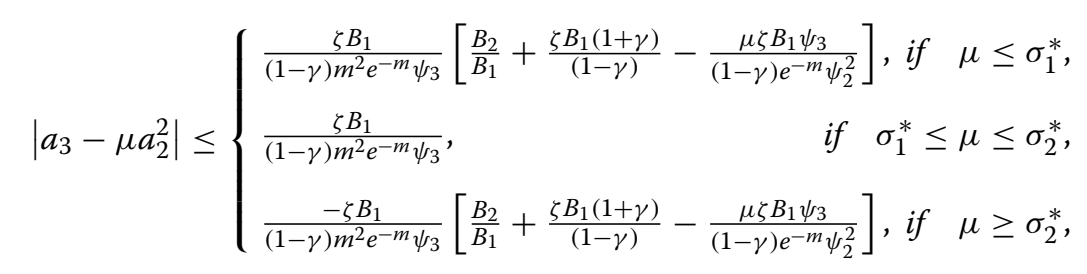

with

$$
\sigma_{1}^{*}=\frac{(1-\gamma) e^{-m} \psi_{2}^{2}}{\zeta B_{1} \psi_{3}}\left[-1+\frac{B_{2}}{B_{1}}+\frac{\zeta B_{1}(1+\gamma)}{1-\gamma}\right]
$$

and

$$
\sigma_{2}^{*}=\frac{(1-\gamma) e^{-m} \psi_{2}^{2}}{\zeta B_{1} \psi_{3}}\left[1+\frac{B_{2}}{B_{1}}+\frac{\zeta B_{1}(1+\gamma)}{1-\gamma}\right]
$$

where $\psi_{k}$ and $k \in\{2,3\}$ are given by (6).

\section{Acknowledgements}

The authors are grateful to the reviewer of this article, that gave valuable remarks, comments, and advices, in order to revise and improve the results of the paper.

\section{Authors' contributions}

All authors jointly worked on the results, and they read and approved the final manuscript.

\section{Funding}

Faculty of Science, Damietta University, New Damietta, Egypt.

\section{Availability of data and materials}

Not applicable.

\section{Competing interests}

The authors declare that they have no competing interests.

\section{Author details}

${ }^{1}$ Department of Mathematics, Faculty of Science, Damietta University, New Damietta 34517, Egypt. ${ }^{2}$ Department of Mathematics, Faculty of Science and Arts in Badaya, Al-Qassim University, Al- Badaya, Al-Qassim, Kingdom of Saudi Arabia. ${ }^{3}$ Faculty of Mathematics and Computer Science, Babeş-Bolyai University, 400084 Cluj-Napoca, Romania.

Received: 22 May 2019 Accepted: 14 October 2019

Published online: 07 November 2019

\section{References}

1. Bulboacă, T.: Differential subordinations and superordinations. Recent Results, House of Scientific Book Publ., Cluj-Napoca (2005)

2. Miller, S. S., Mocanu, P. T.: Differential subordinations: theory and applications, Series on Monographs and Textbooks in Pure and Applied Mathematics, Vol. 225. Marcel Dekker Inc., New York and Basel (2000)

3. Szász, R., Kupán, P. A.: About the univalence of the Bessel functions, Studia Univ. Babeş-Bolyai Math. 54(1), 127-132 (2009)

4. Baricz, Á.: Geometric propertis of generalized Bessel functions. Publ. Math. Debr. 73, 155-178 (2008)

5. Jackson, F. H.: The application of basic numbers to Bessel's and Legendre's functions. Proc. Lond. Math. Soc. 3(2), $1-23(1905)$

6. Selvakumaran, K. A., Szász, R.: Certain geometric properties of an integral operator involving Bessel functions Kyungpook Math. J. 58, 507-517 (2018)

7. Carathéodory, C.: Über den Variabilitätsbereich der Koeffizienten von Potenzreihen, die gegebene Werte nicht annehmen. Math. Ann. 64, 95-115 (1907)

8. Libera, R. J., Zlotkiewicz E.J.: Coefficient bounds for the inverse of a function with derivative in $\mathcal{P}$. Proc. Amer. Math. Soc. 87(2), 251-257 (1983) 
9. Ma, W., Minda, D.: A unified treatment of some special classes of univalent functions. In: Li, Z., Ren, F., Lang, L., Zhang, S. (eds.) Proceedings of the Conference on Complex Analysis, Tianjin, 1992, pp. 157-169. Conf. Proc. Lecture Notes Anal. I, Int. Press, Cambridge, (1994)

10. Porwal, S.: An application of a Poisson distribution series on certain analytic functions. J. Complex Anal., 1-3 (2014). Art. ID 984135. https://doi.org/10.1155/2014/984135

11. Srivastava, D., Porwal, S.: Some sufficient conditions for Poisson distribution series associated with conic regions. Int. J. Adv. Technol. Eng. Sci. 3(1), 229-235 (2015)

\section{Publisher's Note}

Springer Nature remains neutral with regard to jurisdictional claims in published maps and institutional affiliations.

\section{Submit your manuscript to a SpringerOpen ${ }^{\circ}$} journal and benefit from:

- Convenient online submission

Rigorous peer review

- Open access: articles freely available online

- High visibility within the field

- Retaining the copyright to your article

Submit your next manuscript at $\boldsymbol{\triangleright}$ springeropen.com 